\title{
Editorial
}

\section{Fluid Dynamics and Computational Engineering}

\author{
Benchawan Wiwatanapataphee, ${ }^{1}$ Yonghong $\mathrm{Wu}^{2}{ }^{2}$. Ming Tang, ${ }^{3}$ and Shaoyong Lai ${ }^{4}$ \\ ${ }^{1}$ Department of Mathematics, Faculty of Science, Mahidol University, Bangkok 10400, Thailand \\ ${ }^{2}$ Department of Mathematics and Statistics, Faculty of Science and Engineering, Curtin University of Technology, \\ Perth, WA 6845, Australia \\ ${ }^{3}$ Department of Materials Science, Faculty of Science, Kasetsart University, Bangkok 10900, Thailand \\ ${ }^{4}$ Department of Applied Mathematics, Southwestern University of Finance and Economics, Chengdu, Sichuan 610074, China \\ Correspondence should be addressed to Benchawan Wiwatanapataphee; benchawan.wiw@mahidol.ac.th
}

Received 19 October 2014; Accepted 19 October 2014; Published 25 November 2014

Copyright (C) 2014 Benchawan Wiwatanapataphee et al. This is an open access article distributed under the Creative Commons Attribution License, which permits unrestricted use, distribution, and reproduction in any medium, provided the original work is properly cited.

Fluid dynamics is one of the most challenging and exciting research fields today because of the complexity of the subject and the wide range of applications. It concerns the motion of liquids and gases and their interaction with solid bodies. Deep understanding of the full complexity of fluid dynamics is essential for the study and control of many real world problems. The subject of fluid dynamics has been studied intensively worldwide both theoretically and experimentally and has been playing a vital role in the development of new sciences and technologies and optimization of industrial processes and engineering design.

Over the last few decades, intensive research has been carried out worldwide to develop mathematical models and methods for the study of fluid dynamics and their applications to engineering problems. The papers selected for this special issue represent a typical set of contributions in this field of research. Of course, the selected topics and papers are not an exhaustive representation of the recent development in the field. Nevertheless, they provide recent trends and advances of mathematical models and methods for the study of fluid dynamics with different application to engineering and we have the pleasure of sharing these research results with the readers.

This special issue contains twenty-seven papers, covering two main areas: mathematical and numerical analysis and computational model and numerical simulation of engineering problems.

The first set of papers, including four papers, focus on the study of mathematical analysis and numerical scheme for boundary value problems. (i) In the paper titled "The Cauchy problem for a weakly dissipative 2-component Camassa-Holm system," based on the weakly dissipative 2-component Camassa-Holm system, a local well-posedness for the system in Besov spaces is established by using the Littlewood-Paley theory and a priori estimates for the solutions of transport equation.

(ii) In the paper titled "On compact and noncompact structures for the improved Boussinesq water equations," the authors establish analytical expressions of the travelling wave solutions for the nonlinear variants of the generalized Boussinesq water equations with positive and negative exponents.

(iii) In the paper titled "On blow-up structures for a generalized periodic nonlinearly dispersive wave equation," the local well-posedness for a generalized periodic nonlinearly dispersive wave equation is derived under suitable assumptions on initial value, a precise blow-up scenario, and several sufficient conditions.

(iv) In the paper titled "A third order accurate cellwise relaxation implicit discontinuous Galerkin scheme for unstructured hybrid meshes," by using unstructured hybrid meshes, the authors demonstrate a third order accurate cellwise relaxation implicit discontinuous Galerkin (DG) scheme for RANS simulations.

The second set of papers, including twenty-three papers, focus on the development of mathematical models and numerical simulations of various engineering problems.

(i) In the paper titled "Development of a cell-centered Godunov-type finite volume model for shallow water flow based on unstructured mesh," based on the Godunovtype cell-centered finite volume method and the central 
upwind scheme, the authors propose a two-dimensional wellbalanced shallow water model for simulating flows with wetting and drying in a real-life dam-break case with complex topography.

(ii) In the paper titled "Further application of surface capturing method and Cartesian cut cell mesh on hydroelastic water-entry problems of free-falling elastic wedge," the authors investigate the interactions between fluid and elastic structure such as marine lifeboat falling down and ship using a new CFD method on hydroelastic water-entry problem of freefalling elastic wedge.

(iii) In the paper titled "A computational model to assess poststenting wall stresses dependence on plaque structure and stenosis severity in coronary artery," the authors propose a finite element model to study the poststenting hemodynamic stresses and internal stresses over/within the diseased walls of coronary arteries in different states of atherosclerotic plaque.

(iv) In the paper titled "Numerical study on transient heat transfer of a quartz lamp heating system," a finite volume method (FVM) on hybrid grids for radiation and transient conjugate heat transfer is developed and implemented using the fluid flow solver GTEA. The accuracy of the proposed method was tested by the use of a complex geometry furnace with cooling pipes.

(v) In the paper titled "Direct numerical simulation of flow around a circular cylinder controlled using plasma actuators," the authors have performed direct numerical simulation of a flow around a circular cylinder at the Reynolds number of the flow control using plasma actuators under two-dimensional and three-dimensional forcing with different amplitudes.

(vi) In the paper titled "Interaction of water waves and a submerged parabolic obstacle in the presence of a following uniform/shear current using RANS model," based on the Reynolds-Averaged Navier-Stokes (RANS) equations and the free surface deformation, the authors investigate the regular waves propagating over a submerged parabolic obstacle in the presence of a uniform/shear current.

(vii) In the paper titled "Numerical simulation of flow behavior within a venturi scrubber," the three-dimensional numerical simulation of single-phase and two-phase flow (air-water) in a venturi scrubber is presented. The dimensions and model parameters correspond to the industrial applications. The effect of gas velocity for various air-mass flow rate values and five geometries on the pressure drop is analyzed.

(viii) In the paper titled "Numerical investigation of wave slamming of flat bottom body during water entry process," based on the two-phase Reynolds-averaged Naviertype equations, a numerical wave load model is proposed to evaluate hydrodynamic forces exerted on a flat bottom body with a typical deploying condition.

(ix) In the paper titled "Lattice Boltzmann simulation of permeability and tortuosity for flow through dense porous media," based on discrete element method and the Lattice Boltzmann method, the author simulates the fluid flow in a dense porous nonuniform and nonspherical particles and analyzes the relationships among permeability, tortuosity and porosity, and sphere radius.

(x) In the paper titled "Bifurcated SEN with fluid flow conditioners," the performance of a novel design for a bifurcated submerged entry nozzle used for the continuous steel casting is analyzed through CFD numerical simulations of a scaled cold water model.

(xi) In the paper titled "Influences of vorticity to vertical motion of two-dimensional moonpool under forced heave motion," the authors simulate the inviscid-irrotational and viscous flows around a 2D floating rectangular body with a moonpool and the effects of the viscosity and vortex shedding are investigated by changing the gap size, corner shape, and viscosity.

(xii) In the paper titled "Computational analysis of propulsion performance of modified pitching motion airfoils in laminar flow," based on 2D laminar flow with high Reynolds numbers, the effects of shift distance of the pitch axis, frequency, pitching amplitude, pitching profile, and airfoil shape on the thrust generation performance of airfoils are studied.

(xiii) In the paper titled "Flow and noise characteristics of centrifugal fan under different stall conditions," the authors focus on assumption of the flow field for the aerodynamic noise under different conditions. An example of their study is a rotating stall phenomenon in a centrifugal fan.

(xiv) In the paper titled "Kinetic study of nonequilibrium plasma-assisted methane steam reforming," the effect of methane conversion and products yield is investigated using the developed reaction mechanism in plasma-assisted kinetic model and the reaction path flux analysis.

(xv) In the paper titled "Numerical and experimental study of the flow field structure evolution in the circular recess of oil cavity," the authors focus on the laminar radial flow in the oil cavity of heavy-duty computer numerical control machines. The influences of the clearance height, inlet nozzle Reynolds number, and geometrical aspect ratio on flow behaviors are investigated.

(xvi) In the paper titled "Numerical investigation of water droplets shape influence on mathematical modeling results of its evaporation in motion through a high-temperature gas," numerical and experimental investigations of the influence of a single water droplet shape on its evaporation in motion through high-temperature gases have been executed based on the evaporation time, the motion velocity, and the distance passed by various droplet shapes in a high-temperature gases medium.

(xvii) In the paper titled "Fluid-thermal-structural coupled analysis of a radial inflow micro gas turbine using computational fluid dynamics and computational solid mechanics," the authors establish a three-dimensional fluid-thermalstructural coupled analysis for a radial inflow micro gas turbine and investigate the stress distribution of the turbine based on the heat-flow coupling analysis.

(xviii) In the paper titled "Modelling of the tangential strain rate term in the flame surface density transport equation in the context of Reynolds averaged Navier Stokes simulations: a direct numerical simulation analysis," based on the generalised flame surface density (FSD) transport equation in the context of Rey, the authors propose the best way to the modelling of the unresolved dilatation rate and flame normal strain rate contributions. 
(xix) In the paper titled "DEM study of wet cohesive particles in the presence of liquid bridges in a gas fluidized bed," the authors focus on the hydrodynamics of the wet cohesive particles in a gas-fluidized bed. A modified DEMCFD numerical method is developed by compositing an additional liquid-bridge module into the traditional softsphere interaction model.

(xx) In the paper titled "Strength analysis of the carbon fiber reinforced polymer impeller based on fluid solid coupling method," based on the one-way fluid-solid coupling method, the stress distribution of the impeller couple caused by the flow water pressure and rotation centrifugal force of the blade is analyzed.

(xxi) In the paper titled "A nonlinear $k$ - $\varepsilon$ turbulence model applicable to high pressure gradient and large curvature flow," the authors present an improved nonlinear turbulence model to include sensitivity to high pressure gradient flow and large curvature flow.

(xxii) In the paper titled "Computational fluid dynamics modeling three-dimensional unsteady turbulent flow and excitation force in partial admission air turbine," based on the three-dimensional viscous compressible Navier-Stokes equations, the authors employ the renormalization group $k$ $\varepsilon$ turbulence model with finite volume discretization on air turbine with partial admission and analyze various admission rates with full annulus.

(xxiii) In the paper titled "Numerical investigation of a liquid-gas ejector used for shipping ballast water treatment," the authors conduct numerical and experimental researches to obtain the most efficient ballast-water-treatment system under a simple change of nozzle shape.

\section{Acknowledgments}

We would like to thank the authors and reviewers of the papers for the excellent contributions.

Benchawan Wiwatanapataphee Yonghong $\mathrm{Wu}$ I. Ming Tang Shaoyong Lai 


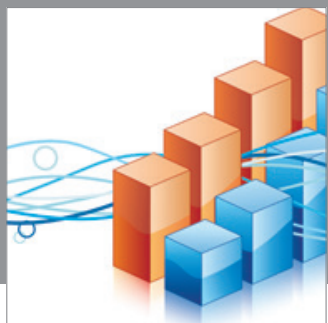

Advances in

Operations Research

mansans

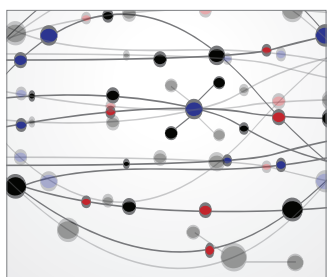

The Scientific World Journal
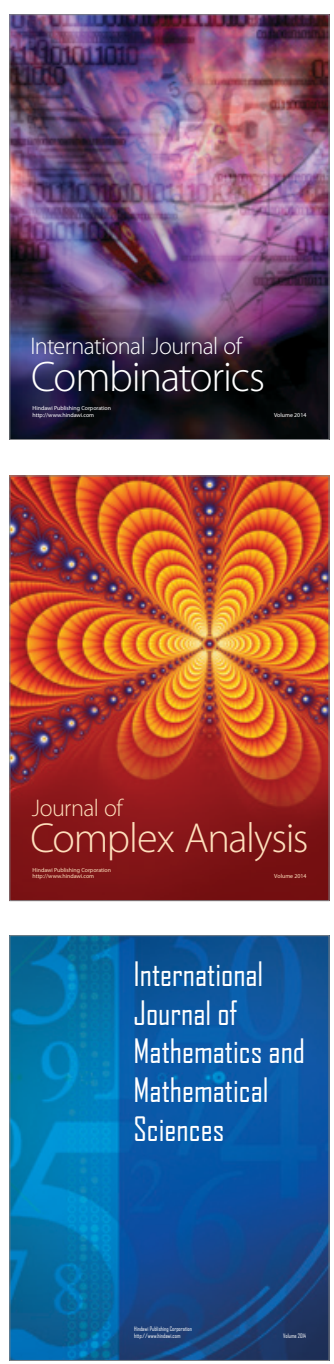
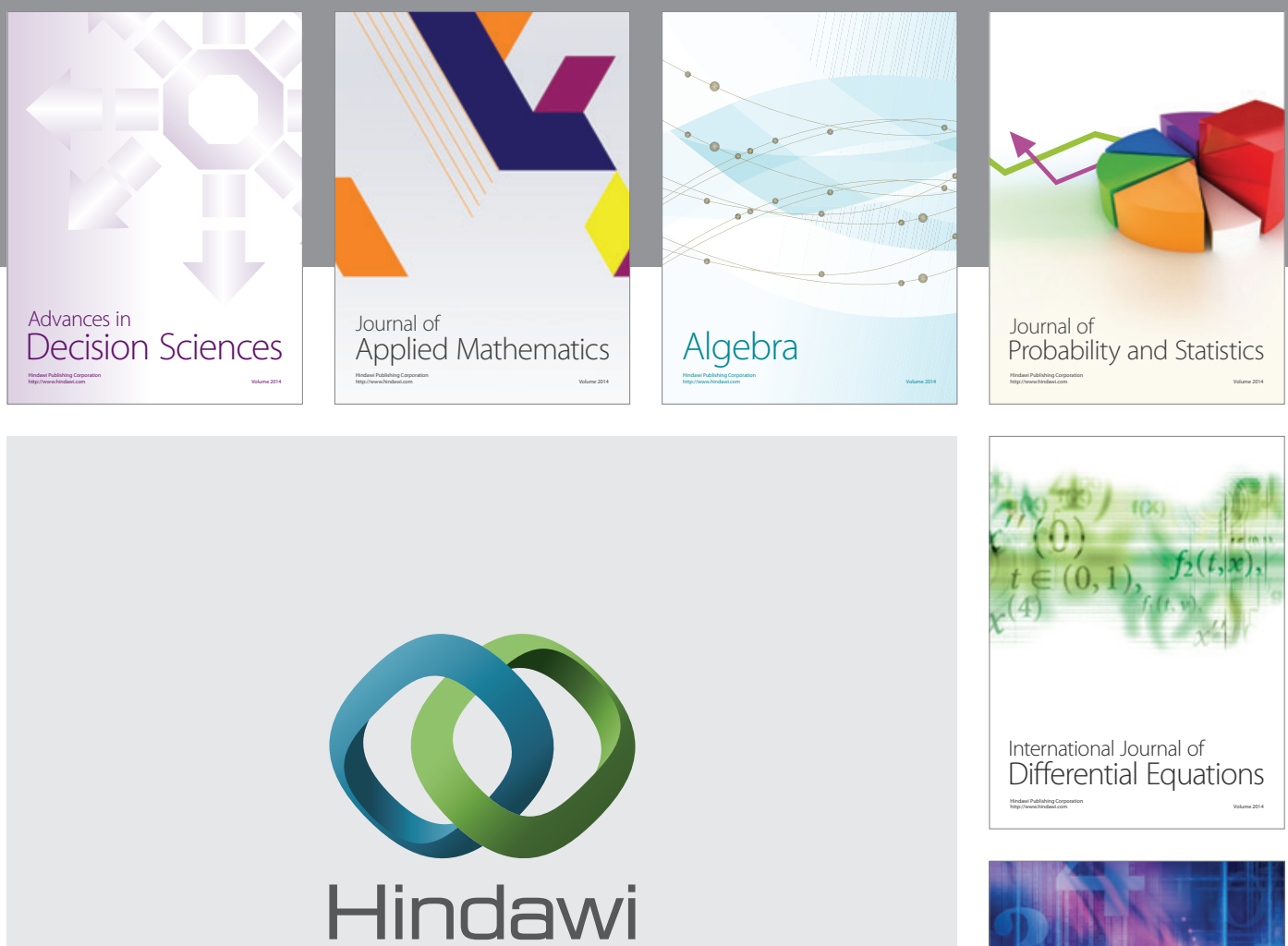

Submit your manuscripts at http://www.hindawi.com
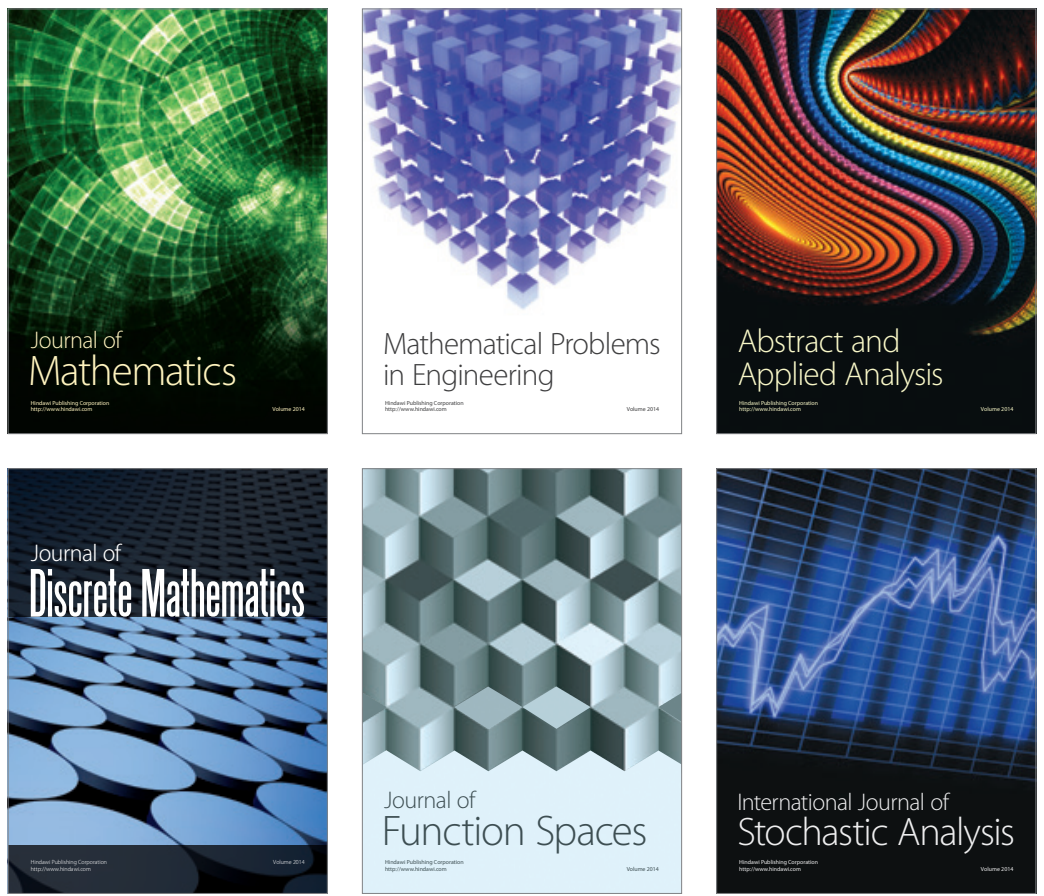

Journal of

Function Spaces

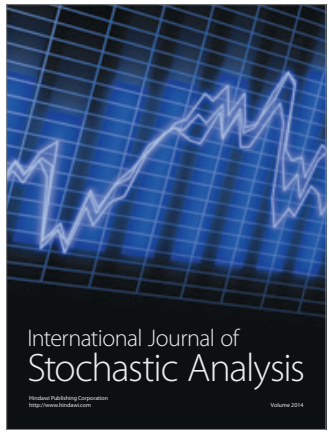

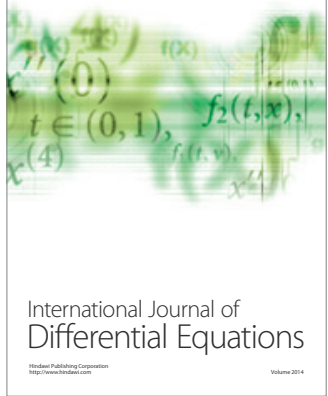
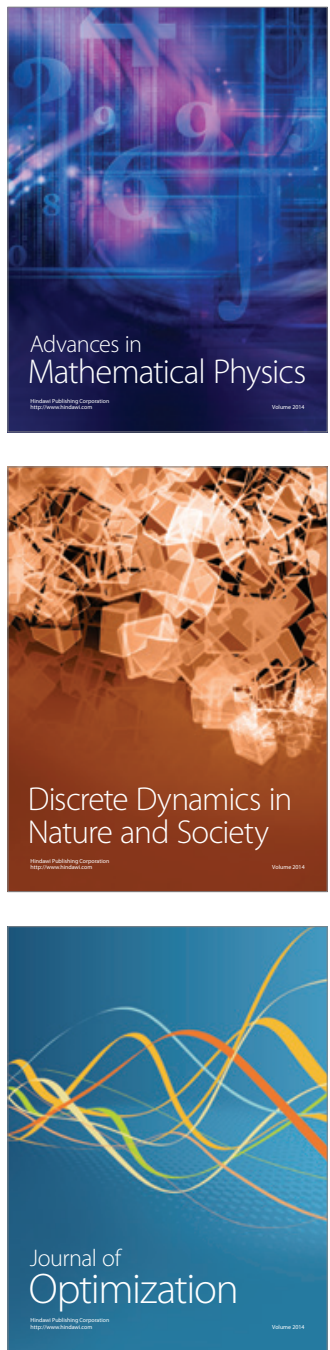